\title{
Gesellschaft „Freunde des Palmengartens e. V.“ Verkürzte Version des Protokolls zur Jahreshauptversammlung vom 25.4.2007
}

\begin{abstract}
Anwesende
Klaus Urban (Vorsitzender, neu), Prof. Dr. Georg Zizka (Vorsitzender, alt), Herta Menk (Schatzmeisterin), Herbert Billensteiner (Schriftführer), Dr. Matthias Jenny (Direktor des Palmengartens), Ilse Henning (Mitarbeiterin der Geschäftsstelle), Anna-Claire Müller (Protokollantin); insgesamt 149 Mitglieder der Palmengarten-Gesellschaft.
\end{abstract}

\section{Ehrungen und Totengedenken}

Totengedenken: Ursula HaCKEnBracht, Maria Klinkert, Ursula Nachtsheim, Gerd Natho-Kochem, Dr. Rudolf Rück, Lieselotte Sperling, Ute von Bauer.

Ehrung für ihre 50-jährige Mitgliedschaft: Hedi und Jürgen W. Rösler, Anni Gnieser, Hanni John.

\section{Jahresbericht des Vorsitzenden}

Als Besonderheit 2006 hob Georg ZizKa das 75-jährige Bestehen der Palmengarten-Gesellschaft hervor. Im Rahmen des Jubiläums fanden verschiedene Aktivitäten der Gesellschaft statt.

29. 5. 2006: Im Haus Rosenbrunn wurde ein Scheck für die Sanierung des Rosengartens durch die Gesellschaft der Oberbürgermeisterin Petra Roth übergeben.

Das Geld kommt der Erneuerung des Rosengartens zu Gute. Der Scheck habe die Stadt Frankfurt zu einer zusätzlichen Förderung ermuntert. Die Arbeiten im Rosengarten sind bereits in vollem Gange. Georg Zizka dankte Klaus Urban für seine Aktivitäten bei der Erneuerung des Rosengartens; Klaus UrbaN bringt seine Kontakte zu Rosenbetrieben ein, beteiligt sich an der Gestaltung des Rosengartens und erarbeitete in Zusammenarbeit mit Anna-Claire Müller den InformationsSchaukasten im Rosengarten.

15.6.2006: Jubiläumsfeier in der Rosenausstellung mit musikalischer Untermalung von Heinz Marx, Ehrenmitglied der Palmengarten-Gesellschaft.

Georg Zizka betonte die hervorragenden Besucherzahlen des Palmengartens, der im Mittelpunkt des Interesses der Gesellschaft steht. Er dankte Matthias Jenny und den Mitarbeiterinnen und Mitarbeitern des Palmengartens für ihre engagierte Arbeit, die sich auch im abwechslungsreichen Ausstellungsprogramm äußerte.

Die Sanierung des Gesellschaftshauses, die auch im Interesse der Palmengarten-Gesellschaft steht, macht Fortschritte. Von der Gesellschaft wurden wie auch in den Vorjahren die Ausstellungen im Palmengarten gefördert, so auch die Ausstellungen, die sich im Jahr 2006 mit dem Thema Südafrika befassten. Ein Novum ist die Förderung der Volontariats-Stelle, die auch 2007 von Anna-Claire Müller besetzt wurde. Traditionell wurde dem Palmengarten von der Gesellschaft eine Spende für das Herbstfest zugeführt, die der Gesellschaft zweckgebunden zuging. Die Gesellschaft möchte stets im Sinne des Palmengartens handeln.

\subsection{Veranstaltungen}

26.4.2006: Vorangegangene Mitglieder-Versammlung.

7. 12.2006: Jubiläumsfeier der Gesellschaft mit etwa 250 Gästen, dazu Gastronomie vom Café Siesmayer im Casino der KfW. Georg Zizka dankte der KfW für die erneute Bereitstellung der Räumlichkeiten und Johnny KLInke für die großzügige Bewirtung.

\subsection{Aktivitäten der Gesellschaft}

Herausgabe und Finanzierung des PalmengartenKalenders 2007, 8 Montags-Vorträge mit jeweils 30 bis 70 Besuchern, 12 Führungen mit jeweils 30 bis 70 Besuchern, 11 Termine zur „Pflanze des Monats" mit jeweils 30 bis 40 Besuchern, 6 Schülerseminare mit jeweils über 30 Besuchern. Thema der Schülerseminare 2006 war „Blüten und Bestäuber“; 2007 lautete das Thema „Bäu- 


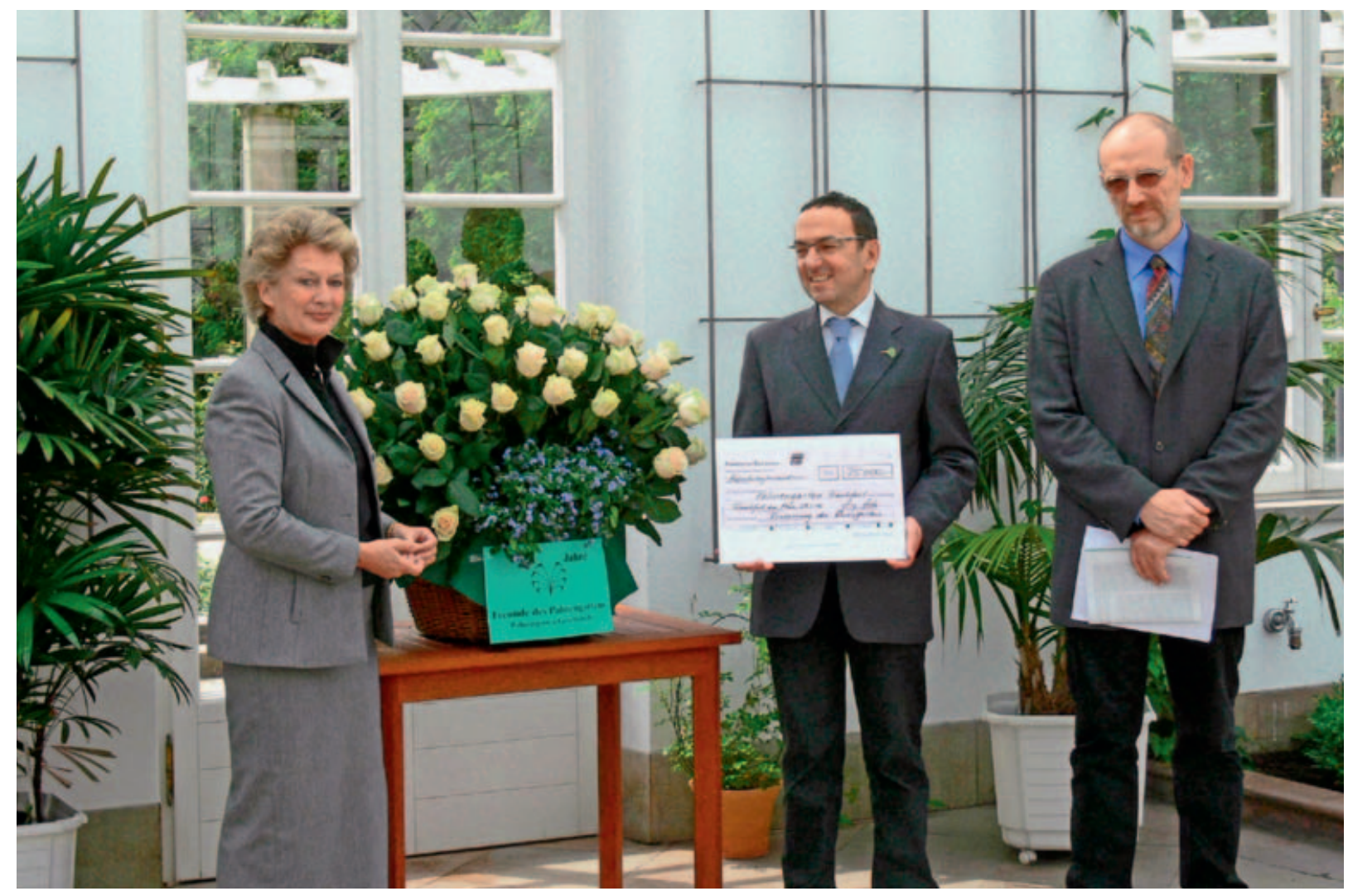

me“. Herta Menk, Herbert Billensteiner und insbesondere DORRIT RöHNERT mit ihrem Helferkreis kümmerten sich 2006 sehr erfolgreich um Infostände und Mitgliederwerbung.

\subsection{Ausflüge, botanische Reisen}

24.6.2006: Tagesfahrt nach Lahnstein und Bad Ems im Lahntal (ausgebucht).

26.8.2006: Tagesfahrt zum Park Schönbusch (Aschaffenburg) und nach Seligenstadt (ausgebucht).

Um die Tagesfahrten kümmert sich nunmehr Frau Dr. Jakoвs (ehemals Frau Dr. WinTER), nachdem diese Reisen bisher lange Jahre von Frau Menk und von dem langjährigen Mitglied Frau Schneider organisiert worden waren. GeOrg ZizKa sprach der anwesenden Frau SCHNEIDER seinen Dank für ihre einstige Arbeit aus.

16.6. bis 22.6.2006: Mehrtagesfahrt mit Ruge-Reisen in die Normandie (ausgebucht).

Abb. 1: Übergabe des Schecks zur Sanierung des Rosengartens: Petra Roth, Matthias Jenny und Georg Zizka.
Mehrtagesfahrt 2007: Schlösser und Gärten an der Loire (ausgebucht).

Tagesfahrten 2007: In den Gail'schen Park und nach Bad Sobernheim (nur noch einige wenige Plätze waren bis zum Zeitpunkt der Versammlung frei).

Das vorgeschlagene Reiseziel Sangerhausen hat sich nach Prüfung als nicht für eine Mehrtagesfahrt geeignet erwiesen. Es wird aber geprüft, ob nicht 2009 eine „Tagesfahrt“ (2-3 Tage) nach Sangerhausen organisiert werden kann, wenn das Interesse an einer solchen Fahrt groß genug sein sollte.

\subsection{Entwicklung der Mitgliederzahlen}

Stand der Mitglieder am 31. 12. 2005: 1488 Zugänge 2006: 275

Abgänge 2006: 114

Stand der Mitglieder am 31. 12. 2006: 1649

Stand der Mitglieder am 23. 04. 2007: 1784

\subsection{Dank}

Dank gilt Herta Menk und Herbert BillenSTEINER für ihre engagierte Arbeit für die Gesellschaft sowie Ditmar Breimhorst, Hilke 
Steinecke, Beate Vaupel und Karin Wittsтоск für Organisation von Vorträgen, Führungen und Veranstaltungen. Dank gilt ebenso den ehrenamtlichen Helfern der Geschäftsstelle; stellvertretend sind hier genannt DORRIT RöHNERT, die sich im Bereich der Mitgliederinformation engagiert, sowie die Kassenprüferinnen Kamilla Nuyken und Helga Urban. Ganz besonderer Dank gilt der Direktion des Palmengartens, Matthias Jenny und Clemens Bayer, für die gute Zusammenarbeit.

\section{Haushalt der Gesellschaft}

Einnahmen durch Beiträge, Spenden, Zinsen: $100737,39 €$

Kosten für Unterhalt der Geschäftsstelle: $21309,25 €$

Förderung des Palmengartens: $48077,13 €$

Kapitalüberschuss: $31351,01 €$

\section{Wahl des Vorsitzenden}

Georg Zizka verkündete seinen Rücktritt vom Amt des Vorsitzenden aus beruflichen Gründen (Überlastung), wie bereits bei seiner Wahl betont worden war. Als Nachfolger wurde KLAUS URBAN vorgeschlagen. Er wurde ohne Gegenstimmen, bei sieben Enthaltungen und mit 142 Ja-Stimmen zum neuen Vorsitzenden der Gesellschaft gewählt. Der neue Vorsitzende überreichte seinem Vorgänger einen Zitronenbaum. [Der neue Vorstand der Palmengarten-Gesellschaft wurde bereits im vorangegangenen PalmengartenHeft 71/1 vorgestellt].

\section{Bericht aus dem Palmengarten von Matthias Jenny}

Der Palmengarten-Direktor betonte die konstante positive Entwicklung der Gesellschaft und dankte Herta Menk für ihr außerordentliches Engagement während der abgelaufenen Amtszeit. Herbert Billensteiner behält im Ruhestand seine Funktion in der PalmengartenGesellschaft bei. Matthias Jenny beglückwünschte KLaus URban zur Wahl zum Vorsitzenden. Es wurde um Verständnis gebeten für die erheblichen Baumaßnahmen im Palmenhaus, Gesellschaftshaus und Rosengarten; gleichzeitig wurde der Gesellschaft für ihre stets unterstützenden Maßnahmen gedankt.

\section{Anschriften der Autorinnen und Autoren}

Kurt Baumann, Euckenstr. 13a, 65929 Frankfurt

Josef Bogner, Augsburger Str. 43 A, 86368 Gersthofen

Dr. Gennady Firsov, Korablestroitelei Street, House 42, Flat 403, Saint-Petersburg, 199155, Russia,

E-Mail: Gennady_firsov@mail.ru

Hans Grasmück, Hohe Str. 36, 63069 Offenbach, E-Mail: hans.grasmueck@arcor.de
Athina Hatziathanasiadou,

Botanischer Garten Stavroupoli, Griechenland, E-Mail: botanic.gard@stavroupoli.gr

Dr. Karin Steinecke, Feldhäuser Str. 76, 28865 Lilienthal, E-Mail: steinecke@web.de

Herwig Zahorka, JI Bondongan, Puri Mas C27, Bogor, 16131, Indonesien, E-Mail: zahorka@indo.net.id

Nicht genannte Autorinnen und Autoren gehören dem Palmengarten an. 